\title{
Whether Slow-Pull-Back or Standard Suction, Optimal Histologic Core Procurement Is Most Important Factor Rather than Blood Contamination or Cellularity in Endoscopic Ultrasound-Guided Tissue Acquisition of Pancreatic Solid Tumor
}

\author{
Se Woo Park \\ Division of Gastroenterology, Department of Internal Medicine, Hallym University College of Medicine, Hwaseong, Korea
}

Corresponding Author

Se Woo Park

ORCID https://orcid.org/0000-0003-1603-7468

E-mail britnepak@hallym.or.kr
See "A Meta-Analysis of Slow Pull versus Suction for Endoscopic Ultrasound-Guided Tissue Acquisition" by Yousuke Nakai, et al. on page 625, Vol. 15, No. 4, 2021
Endoscopic ultrasound (EUS)-guided tissue acquisition (TA) of pancreatic solid tumor has been considered a standard approach for establishment of an accurate diagnosis and personalized therapeutic plan with rare adverse events. However, it has still limitations such as low negative predict value which cannot guarantee no malignancy in final diagnosis in cases that were initially classified as negative results despite recent innovation of newly designed needle and development of new endoscopic techniques. Recently, histologic core by EUS-guided fine needle biopsy (FNB) can facilitate an accurate differential diagnosis of various pancreatic solid tumors by specialized immunohistochemistry (IHC) staining in the current personalized medicine era. ${ }^{1}$ Despite this advancement of EUS-TA of pancreatic solid tumors, many technical aspects still require clinical standardization. Among these, the application of suction on the needle mount can be theoretically a standard practice based on the hypothesis that negative pressure by syringe would increase cellularity and further diagnostic accuracy. More recently, alternative capillary-pressure technique by pulling the needle stylet slowly during toand-pro movement has been introduced. This technique called "capillary aspiration" or "slow-pull-back method" can result in improved diagnostic accuracy by better cellularity and lesser blood contamination.

Despite the mainstream nature of the practice, a widespread approach between standard suction and new slowpull-back method has not yet been established. Therefore, clinical practice surrounding suction during EUS-TA including EUS-guided fine needle aspiration (FNA) and FNB vary considerably among training system of each institution and individual experience of physicians. Recent clinical guideline ${ }^{2}$ suggests that routine application of standard suction can be recommended in tumors with poor cellularity such as chronic pancreatitis or ductal adenocarcinoma, while it cannot be recommended in tumors which may contain rich cellularity with high vascularity or extensive necrosis. Moreover, the slow-pull-back method may be more effective regarding a procurement of adequate histologic core with fewer needle passes.

In the previous issue of Gut and Liver, Nakai et al. ${ }^{3}$ addressed the very topical subject matter of the diagnostic yield of the slow-pull-back method and compared them with those of the standard suction. The authors then made an effort to obtain consensus by using a systematic review and meta-analysis. However, there is a non-negligible heterogeneity regarding to study type (seven randomized controlled trials [RCTs], four prospective studies, and six retrospective studies), thus it can lead to significant bias in the process of summation for data of included studies. In performing a meta-analysis, an investigator should select multiple scientific studies addressing the same question, with each individual study reporting measurements that are expected to have some degree of error. ${ }^{4}$ In other words, the simultaneous summation of data from RCTs and retrospective studies may occasionally lead to different conclu- 
sions either positive or negative results because the study design as well as specific endoscopic techniques and devices were not controlled uniformly in retrospective studies. In same conception, there are significant heterogeneities regarding the definition of cellularity and blood contamination across the included studies although authors used a random-effects model and performed subgroup analysis to account for any between study variability. They may be due to the heterogeneous endoscopic devices (diameter of needle [ 22 gauge or 25 gauge], type of needle [FNA or FNB], or amounts of suction [10 mL, $20 \mathrm{~mL}$, or higher]) and different procedural protocols across the studies. Thus, clinicians should cautiously interpret the results of this meta-analysis when applied to clinical practice.

Recent randomized trial ${ }^{5}$ comparing suction and nosuction for pancreatic tumor reported that the application of suction resulted in higher cellularity but higher blood contamination. Therefore, suction can decrease the quality of sample due to the higher risk of blood contamination. In contrast to standard suction method, slow-pull-back method minimizes negative pressure by withdrawal the stylet continuously and slowly. ${ }^{6}$ In a recent $\mathrm{RCT}^{7}$ comparing three groups (the slow-pull-back vs standard suction vs non-suction method), the standard suction method had a higher blood contamination rate but did not result in increased histologic core procurement rate. Furthermore, the authors reported that the slow-pull-back method provided greater cellularity with lesser blood contamination compared with the other method, although the diagnostic adequacy based on histologic core tissue for malignancy was not differ between the groups. In addition, slow-pullback method can provide more expeditious results based on lesser number of cytologic slides while guaranteeing results of higher cellularity and lower blood contamination in recent study. ${ }^{8}$

However, most important variable in recent EUS-TA is quality of histologic sampling not cellularity or blood contamination in the personalized medicine era. Better histologic and molecular evaluation of the pancreatic tumor can be expected in a tissue sample containing histologic core. In evaluation of histologic sample even in sample by cell block method, the cellularity or blood contamination cannot be an issue for determining of malignancy. Generally, liquid-based cytology, cell block, and histologic samples can all be used for IHC. ${ }^{9}$ Furthermore, IHC as well as molecular assays can play critical roles in differentiating benign or malignant lesions and the tracing the origin of metastatic tumors of the pancreas. Recent study evaluated the quantity of histologic sample by the computer-assisted measurement of the core fragment surface area. They reported that the quality of histologic sample had a strong correlation with quantity of sample measured by computer-assisted system, therefore, the larger histologic core specimens were able to improve the diagnostic accuracy. ${ }^{10}$

In conclusion, this meta-analysis has revealed that slowpull-back method had several advantages of lesser blood contamination and better diagnostic accuracy. In my view, whether slow-pull-back or standard suction is better regarding blood contamination, endoscopists should pay attention to procurement of optimal histologic core based on special pathologic evaluation such as IHC not cytologic evaluation in the personalized medicine era.

\section{CONFLICTS OF INTEREST}

No potential conflict of interest relevant to this article was reported.

\section{ORCID}

Se Woo Park https://orcid.org/0000-0003-1603-7468

\section{REFERENCES}

1. Park SW, Chung MJ, Lee SH, et al. prospective study for comparison of endoscopic ultrasound-guided tissue acquisition using 25- and 22-gauge core biopsy needles in solid pancreatic masses. PLoS One 2016;11:e0154401.

2. Chung MJ, Park SW, Kim SH, et al. Clinical and technical guideline for endoscopic ultrasound (EUS)-guided tissue acquisition of pancreatic solid tumor: Korean Society of Gastrointestinal Endoscopy (KSGE). Gut Liver 2021;15:354374.

3. Nakai Y, Hamada T, Hakuta R, et al. A meta-analysis of slow pull versus suction for endoscopic ultrasound-guided tissue acquisition. Gut Liver 2021;15:625-633.

4. Walker E, Hernandez AV, Kattan MW. Meta-analysis: its strengths and limitations. Cleve Clin J Med 2008;75:431-439.

5. Lee JK, Choi JH, Lee KH, et al. A prospective, comparative trial to optimize sampling techniques in EUS-guided FNA of solid pancreatic masses. Gastrointest Endosc 2013;77:745751.

6. Matsubayashi H, Matsui T, Yabuuchi Y, et al. Endoscopic ultrasonography guided-fine needle aspiration for the diagnosis of solid pancreaticobiliary lesions: clinical aspects to improve the diagnosis. World J Gastroenterol 2016;22:628640.

7. Lee KY, Cho HD, Hwangbo Y, et al. Efficacy of 3 fine-needle biopsy techniques for suspected pancreatic malignancies in 
the absence of an on-site cytopathologist. Gastrointest Endosc 2019;89:825-831.

8. Bor R, Vasas B, Fábián A, et al. Prospective comparison of slow-pull and standard suction techniques of endoscopic ultrasound-guided fine needle aspiration in the diagnosis of solid pancreatic cancer. BMC Gastroenterol 2019;19:6.

9. Matsubayashi H, Sasaki K, Ono S, et al. Pathological and molecular aspects to improve endoscopic ultrasonography- guided fine-needle aspiration from solid pancreatic lesions. Pancreas 2018;47:163-172.

10. Karsenti D, Palazzo L, Perrot B, et al. 22G Acquire vs. 20G Procore needle for endoscopic ultrasound-guided biopsy of pancreatic masses: a randomized study comparing histologic sample quantity and diagnostic accuracy. Endoscopy 2020;52:747-753. 